\title{
Analysis of a pharmacist-led adverse drug event management model for pharmacovigilance in an academic medical center hospital in China
}

This article was published in the following Dove Press journal:

Therapeutics and Clinical Risk Management

Wei He

Difei Yao

Yangmin Hu

Haibin Dai

Department of Pharmacy, Second Affiliated Hospital, Zhejiang University School of Medicine, Hangzhou 310009 , China

Correspondence: Haibin Dai Department of Pharmacy, Second Affiliated Hospital, Zhejiang University School of Medicine, 88 jiefang Road, Hangzhou 310009, China

Tel +8657 |8 778389 |

Fax +86 57 I8 778 389।

Email haibindai@zju.edu.cn
Background: Spontaneous reporting of adverse drug events (ADEs) has long been the cornerstone of pharmacovigilance. Medical institutions in China have been a major source of $\mathrm{ADE}$ case reports, but the proportion of reports from tertiary hospitals is low due to the serious underreporting of case reports. The same problem existed in the Second Affiliated Hospital of Zhejiang University School of Medicine (SAHZU).

Objective: In order to increase the number of ADE reports and promote hospital pharmacovigilance, SAHZU's clinical pharmacists established a pharmacist-led ADE management model. The aim of this paper is to introduce this management model and explore the advantages and disadvantages of the model.

Methods: Pharmacist-led ADE management model was gradually formed from 2015 to 2017 in the SAHZU. This "pharmacist-led" model is reflected not only in the fact that clinical pharmacists are the main reporters of SAHZU's ADEs but also in that they are the main groups to analyze and manage ADE and drug errors. The sources of ADEs reported by clinical pharmacists mainly include pharmacy rounds, ADE-related pharmacist consultations, centralized monitoring, ADE warning signal analysis, newly introduced drug evaluations, and drug safety research.

Results: A total of 533 ADEs were reported by SAHZU to China's spontaneous reporting system (SRS) in 2017, while the data in 2012 was 177, with an increase by $201 \%$. In 2012, the proportion of "new" and "serious" reports was 16.4\%. The proportions during the period from 2015 to 2017 were $41.4 \%, 60.8 \%$, and $52.2 \%$, respectively, which were statistically significant compared with the proportion in 2012. The proportion of ADEs reported by clinical pharmacists during the period from 2014 to 2017 were $51.5 \%, 57.3 \%, 68.8 \%$, and $90.8 \%$, respectively, which were statistically significant compared with the proportion in $2013(P<0.05)$. There was a correlation between the proportions of severe ADEs and the proportion of ADEs reported by clinical pharmacists $(r=0.873$, $P=0.023$ ). Four hundred eighty four ADE cases reported by clinical pharmacists to China's SRS in 2017 were mainly found in rounds of clinical pharmacists (74.17\% [359/484]).

Conclusion: The pharmacist-led pharmacovigilance working model significantly increased the quantity and quality of ADE reporting in SAHZU and promoted pharmacovigilance. This model is worth developing in Chinese tertiary hospitals and the following hospitals, where the physicians working there spend little time and energy on ADE reporting or the cost of physicians is high, while the clinical pharmacist team has strong professional skills.

Keywords: tertiary hospitals, new adverse drug events, serious adverse drug events, clinical pharmacist, active surveillance

\section{Introduction}

In 2002, the WHO formally extended the definition of pharmacovigilance to include the scientific activities of finding, assessing, understanding, and preventing drug-related BY NC and incorporate the Creative Commons Attribution - Non Commercial (unported, v3.0) License (http:///creativecommons.org/licenses/by-nc/3.0/). By accessing the work you
hereby accept the Terms. Non-commercial uses of the work are permitted without any further permission from Dove Medical Press Limited, provided the work is properly attributed. For permission hereby accept the Terms. Non-commercial uses of the work are permitted without any further permission from Dove Medict
for commercial use of this work, please see paragraphs 4.2 and 5 of our Terms (https://www.dovepress.com/terms.php). 
adverse effects or any other drug-related safety issue. ${ }^{1}$ Adverse drug events (ADEs) are an important part of the pharmacovigilance system. ${ }^{2}$ China National Center for Adverse Drug Reaction Monitoring (CNCAM) was established in 1989, and China has a online spontaneous reporting system (SRS) with a four-level network that consists of one national center, 34 provincial centers, and $>400$ municipal centers. $^{3}$ The definition of adverse drug reactions is strict, but China encourages reporting of all possible drug-related adverse events to SRS. Due to differences in the national management system, the sources of ADE in China are different from those in the US. According to relevant literature, from the end of 2000 to the end of 2001, most reports, as many as $96.43 \%$, received by the US FDA's adverse event reporting system came from manufacturers. In addition, US consumers are also one of the major sources of ADEs., In contrast, China's annual report on ADE monitoring (2016) showed that $85.6 \%$ of ADEs come from medical institutions in China, but the proportion of reports in tertiary hospitals is low due to underreporting. According to the "Hospital Classification Management Measures" promulgated by the Ministry of Health of the People's Republic of China, Chinese hospitals are divided into three grades. The tertiary hospitals, which should have more than 501 beds, provide high-level specialist health services and performs higher education and research tasks in several regions. The data showed that on average, only 37.4 ADE reports were submitted by each tertiary hospital in 2015. ${ }^{6}$ The Second Affiliated Hospital of Zhejiang University (SAHZU), Hangzhou, China, is a large, integrated, research-based tertiary hospital integrating medical treatment, teaching, and research. At present, SAHZU has a total of 3,200 beds, and the number of hospitalizations in 2017 was nearly 154,000. However, the number of ADE reports per year before 2011 was less than 100. In recent years, the China Food and Drug Administration (CFDA) has promoted the exploratory use of active pharmacovigilance by the scientific community and medical institutions. The pharmacovigilance system in China (China Hospital Pharmacovigilance System [CHPS]) was launched online in 2017. SAHZU is a provincial sentinel, and has applied to become a national sentinel. In order to increase the number of $\mathrm{ADE}$ reports and promote hospital pharmacovigilance, SAHZU's clinical pharmacists gradually established a pharmacist-led ADE management model. The aim of this paper is to introduce this management model, explore the pros and cons of pharmacovigilance in medical institutions led by clinical pharmacists, and investigate ideas for further development.

\section{Methods}

The pharmacist-led ADE management model was studied by referencing and learning from: 1) information collected from scientific papers and the websites of CNCAM and the CFDA, 2) information available on China's SRS and active pharmacovigilance system, 3) China's annual report on ADE monitoring, and 4) data obtained from SAHZU hospital internal reporting system and internal information.

\section{Overview of ADE management model of SAHZU}

Like most medical institutions at home and abroad, previous ADE management at SAHZU used a simple voluntary reporting model. Under the leadership of the hospital's ADE management team, the ADE management pharmacist received ADE reports through telephone or face-to-face communication, and excluded unqualified, inaccurate, or near misses and other non-ADE reports and then submitted the report to the China's SRS after quality checking. ADE management pharmacist is a senior clinical pharmacist and trained by ADE monitoring center. This type of monitoring, which only collects spontaneous reports, has various drawbacks, such as omission, underreporting, duplicate cases, incalculable incidence, and information bias in practical work, which hinder the generation and evaluation of adverse event signals. ${ }^{7,8}$ Before 2012, the number of ADEs reported in SAHZU was less than 100 cases per year, and the least that was observed was less than 10 cases in 1 year. To encourage and promote the medical staff to report ADEs, the ADE management team took various measures, including strengthening information and education in key departments, establishing an internal hospital network direct reporting system, and providing rewards for reporting.

The number of reported in-hospital cases rose from 65 in 2011 to 394 in 2014, but reports from physicians have always been fewer (the annual reported number remains at $10-20$ cases). Since 2015 , to improve the availability of reported ADEs, China raised the requirements for the proportion of "new" and "serious" ADE reports, ${ }^{9}$ but this proportion could not be met with the past model of spontaneous reporting. The proportion of "new" and "serious" ADE reports from SAHZU reported to the China's SRS in 2014 was $19.0 \%$, while the country's requirement was 33\%. Beginning in 2015, SAHZU adjusted the reporting policy of ADE and encouraged medical personnel to report "new" and "serious" ADE reports, especially "serious" ADE reports, due to China's clear requirement for the "new" and "serious" ADE reporting ratio. 
Because more ADEs can be identified through the rational use of drug and safety management, clinical pharmacists began to shift their focus to the collection and reporting of ADEs for hospital drug alerts and adopted a variety of active monitoring methods to make the ADE collection more scientific and effective, gradually forming a pharmacist-led pharmacovigilance model of operation. This "pharmacistled" model is reflected not only in the fact that clinical pharmacists are the main reporters of SAHZU's ADEs but also in that they are the main groups to analyze and manage ADE and drug errors. The sources of ADEs reported by clinical pharmacists mainly include timely reporting of ADEs after pharmacy rounds, ADE-related pharmacist consultations, centralized monitoring of key department and key drugs, early signals analysis of safety issues, drug quality management and procedural improvements, newly introduced drug evaluations and drug safety research. SAHZU established liaison staff in various departments for hospital quality management needs. Clinical pharmacists and key liaison people, such as radiology department, infusion rooms, etc., communicate regularly with each other. Specific drug events are discussed and processed with the relevant department liaisons in a timely manner. Through strengthening communication with clinical departments, clinical pharmacists can better understand the medication characteristics and common ADEs in different departments, which not only improve clinical pharmacists' practical processing ability but also solve more practical problems for clinical practice and improve the rationality of clinical medication and the clinician's recognition.

\section{Various pharmaceutical services related to active pharmacovigilance Centralized monitoring}

Post-marketing drug surveillance for ADEs has typically relied on spontaneous reporting. ${ }^{10}$ Centralized monitoring of key drugs, key departments, and key ADEs will help increase the discovery rate of ADEs and strengthen the safety of hospital medication. Key drugs generally refer to commonly used drugs with high incidence of ADE, such as specific antibacterial drugs, anticoagulant drugs, and glucocorticoids. Key departments generally refer to departments with high incidence of ADE or prone to severe ADE, such as department of radiology, infusion, and anesthesia. Key ADEs generally refer to the ADEs which can lead to common drug-induced diseases, such as drug eruption, drug-induced liver damage, and drug-induced renal damage. Clinical phar- macists can obtain ADEs information by regularly searching for relevant diagnoses in the hospital information system. Drugs, departments, and ADEs for centralized monitoring can be adjusted according to the needs of hospital drug safety management.

\section{Verification underreporting}

Underreporting is the biggest flaw of the spontaneous reporting system. In order to reduce underreporting and improve the attention of medical staff to report ADE, clinical pharmacists randomly selected dozens of electronic medical records from the discharged patients every month to check whether there were ADEs that had not been reported. In cases where serious ADEs are not reported, the hospital management department would punish relevant medical staff.

\section{Newly introduced drug evaluations}

A 6-month evaluation is required for the newly introduced drugs of SAHZU, including efficacy, adverse events, and rational use. The efficacy of these drugs is generally assessed by physicians, and adverse events and rational use are evaluated by clinical pharmacists. If there is a major safety incident or significant unreasonable use during the newly introduced drug evaluation period, it will be submitted to the Hospital Pharmacy Management Committee to determine whether it needs to be rectified or eliminated.

\section{Safety research}

Recently, Chinese regulatory agencies have turned more attention to active pharmacovigilance and drug safety research. Strengthening cooperation between multiple hospitals and monitoring centers can avoid information isolation and promote better and wider use of existing pharmacovigilance data. Clinical pharmacists at tertiary hospitals with research tasks are one of the best people for drug safety research. SAHZU is conducting a drug safety research project and also participating as a key member in the safety research project of provincial and municipal monitoring centers.

\section{Early signals of safety issues and continuous process improvement}

Spontaneous reporting of suspected ADEs has long been the cornerstone of pharmacovigilance for the identification of early signals of problems of safety related to the use of medicines. ${ }^{11,12}$ Beginning in 2015, ADE management pharmacists began to use the ADE data they had received 
to identify early signals of safety issues related to drug use, including frequent adverse reactions to a certain drug in the short term, high incidence ADE in specific departments, high incidence ADE among similar patients (such as age, region, disease, etc), and safety signals from "new" and "severe" ADE. After screening and evaluation, these signals can indicate potential safety issues in drug use, personnel operations, and hospital processes, and provide evidence and direction for the continuous improvement of hospital drug safety management.

\section{Statistical analyses}

Chi-squared and Fisher's tests were used for testing occurrence rate differences between the two groups. Pearson's correlation coefficient test was used to evaluate the relationship. All statistical analyses were performed using SPSS software (v 13.0). A $P$-value $<0.05$ was considered to be statistically significant.

\section{Results}

\section{Number of ADE reports and proportion of "new" and "serious" ADE reports}

The number of ADE reports submitted by SAHZU to China's SRS exhibited an increasing trend during 2012-2014. There was a decline in the number of reports in 2015. Then, the number of ADE reports exhibited another increasing trend during 2015-2017, and reached a record high in 2017. In 2012, the proportion of "new" and "serious" reports was $16.4 \%$. There was no statistically significant difference in this proportion during 2012-2014 ( $P>0.05)$. However, the proportions during the period from 2015 to 2017 were $41.4 \%$, $60.8 \%$, and $52.2 \%$, respectively, which were statistically significant compared with the proportion in 2012 (Figure 1).
The proportion of ADE reported by clinical pharmacists exhibited an increasing trend during 2013-2017. The proportions during the period from 2014 to 2017 were $51.5 \%, 57.3 \%$, $68.8 \%$, and $90.8 \%$, respectively, which were statistically significant compared with the proportion in $2013(P<0.05)$. The proportions of "new and severe ADEs" and "severe ADEs" exhibited similar increasing trends during 2015-2017, which were statistically significant compared with the proportion in $2012(P<0.05)$. There was a correlation between the proportions of severe ADEs and the proportion of ADEs reported by clinical pharmacists $(r=0.873, P=0.023)$, and this relation was statistically significant (Figure 2 ).

It is worth noting that the ADEs submitted to China's SRS were all reviewed and screened by the management pharmacists. The 2017 data showed that 707 cases of ADE were received by the SAHZU hospital internal reporting system. When the ADEs were managed by pharmacists to remove non-ADEs, near misses, and very mild common ADE reports, the number of ADEs submitted to China's SRS was 533. After such review and preliminary screening work, the proportion of pharmacist-derived reports rose from $70.0 \%$ to $90.8 \%$, while the proportion of "new" and "severe" ADEs increased from $39.3 \%$ to $52.2 \%$.

\section{ADE sources reported by clinical pharmacists}

Further analysis of 484 ADE cases reported by clinical pharmacists to China's SRS in 2017 showed that these were mainly found in rounds of clinical pharmacists (74.17\% [359/484]) (Figure 3). All of these ADE reports can be considered as related to pharmacovigilance, of which active monitoring includes centralized monitoring (11.98\% [58/484]), verification underreporting (1.24\% [6/484]), newly introduced drug

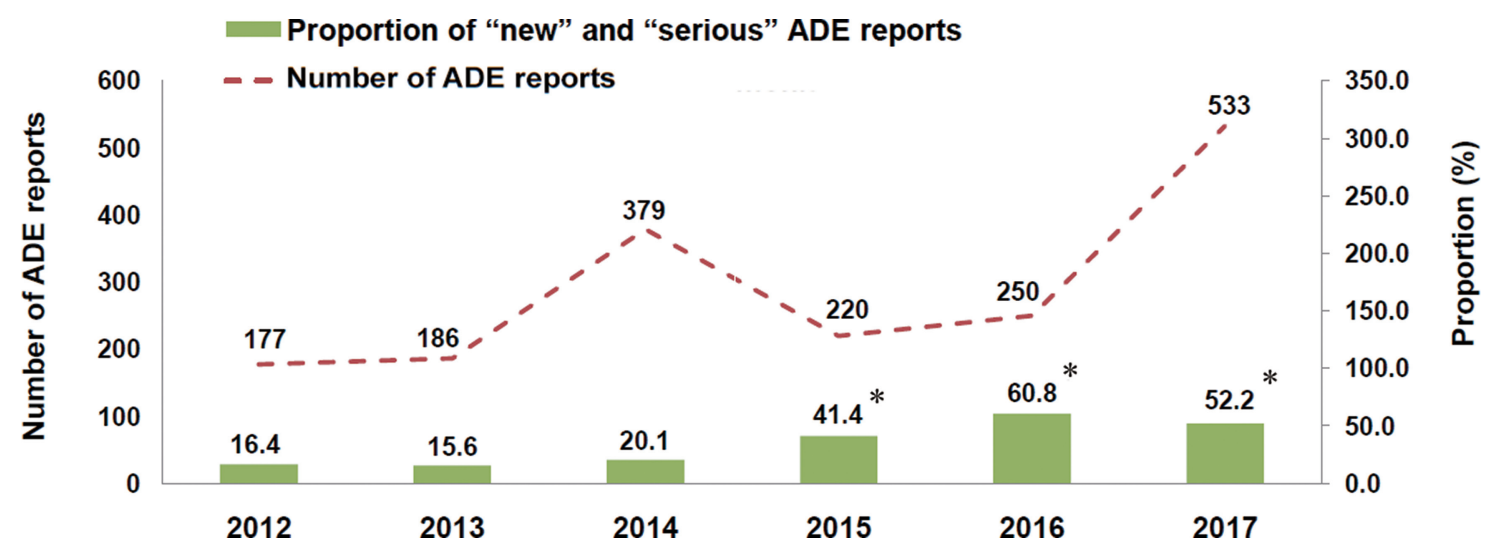

Figure I Numbers of ADE reports submitted by SAHZU to China's SRS and proportion of "new" and "serious" ADE reports.

Notes: Proportion of "new" and "serious" ADE reports (\%). $* P<0.05$ (compared with data of 20I2).

Abbreviations: ADE, adverse drug event; SAHZU, the Second Affiliated Hospital of Zhejiang University; SRS, spontaneous reporting system. 


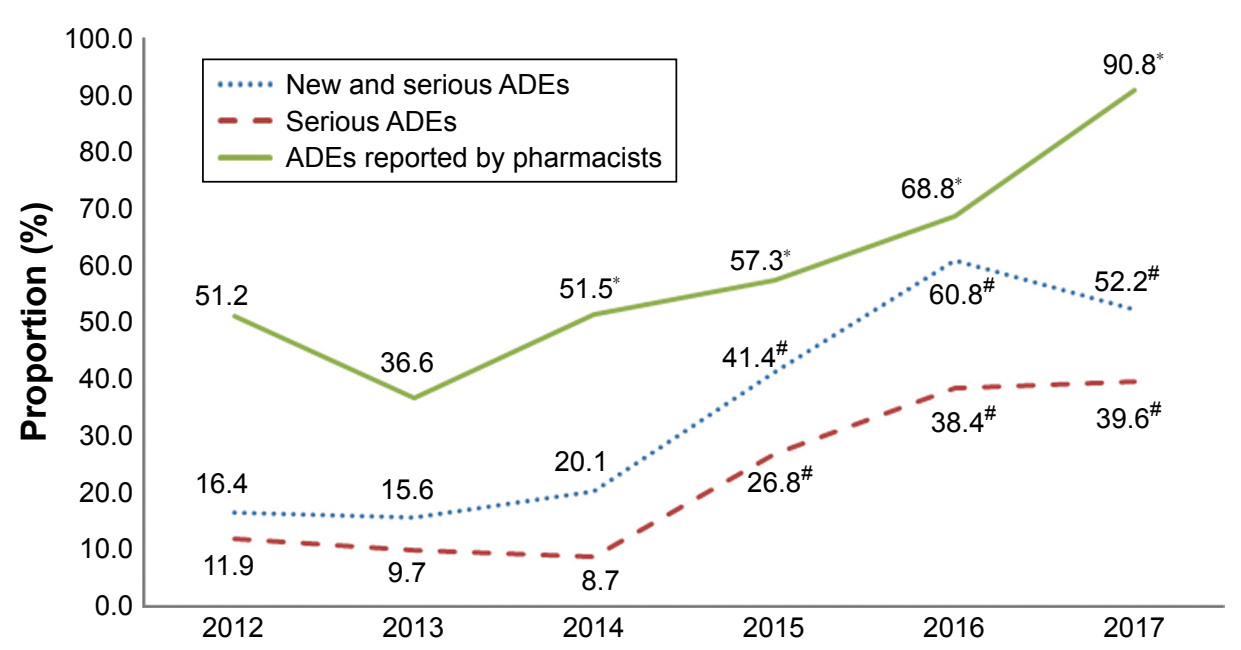

Figure 2 Proportions of "new" and "serious" ADE reports and "serious" ADE reports submitted by SAHZU to China's SRS and the proportion of reports submitted by pharmacists during 2012-2017.

Notes: Proportion of reports submitted by clinical pharmacists (\%), ${ }^{*} P<0.05$ (compared with data of 20I3). Proportions of "new" and "serious" ADE reports and "serious" ADE reports submitted by SAHZU to China's SRS (\%), ${ }^{P}<0.05$ (compared with data of 20I2).

Abbreviations: ADE, adverse drug event; SAHZU, the Second Affiliated Hospital of Zhejiang University; SRS, spontaneous reporting system.

evaluations $(1.03 \%[5 / 484])$, and safety research $(2.07 \%$ [10/484]), which comes to a total of $16.32 \%$.

\section{Discussion}

Data from 2012 to 2017 showed that the number of ADE reports from SAHZU has shown two upward trends in 2014 and 2017 , respectively, and a decline in 2015. This phenomenon may be explained by the following four factors. The first factor was the Joint Commission International (JCI) accreditation. SAHZU successfully passed the JCI accreditation as an academic medical center hospital on February 24,
2013. A working group composed of nurses, pharmacists, information engineers, physicians, and administrators was established to implement multifaceted interventions at the individual, organizational, and policy levels to reduce adverse events in the post-JCI accreditation era. The second factor was the change in the number of clinical pharmacists. In 2012, SAHZU had only six full-time clinical pharmacists, which increased to 12 in 2014 and further increased to 20 in 2017. The two time points of increasing number of clinical pharmacists are consistent with the peak time of the number of ADE reports. The third factor was that starting in 2017,

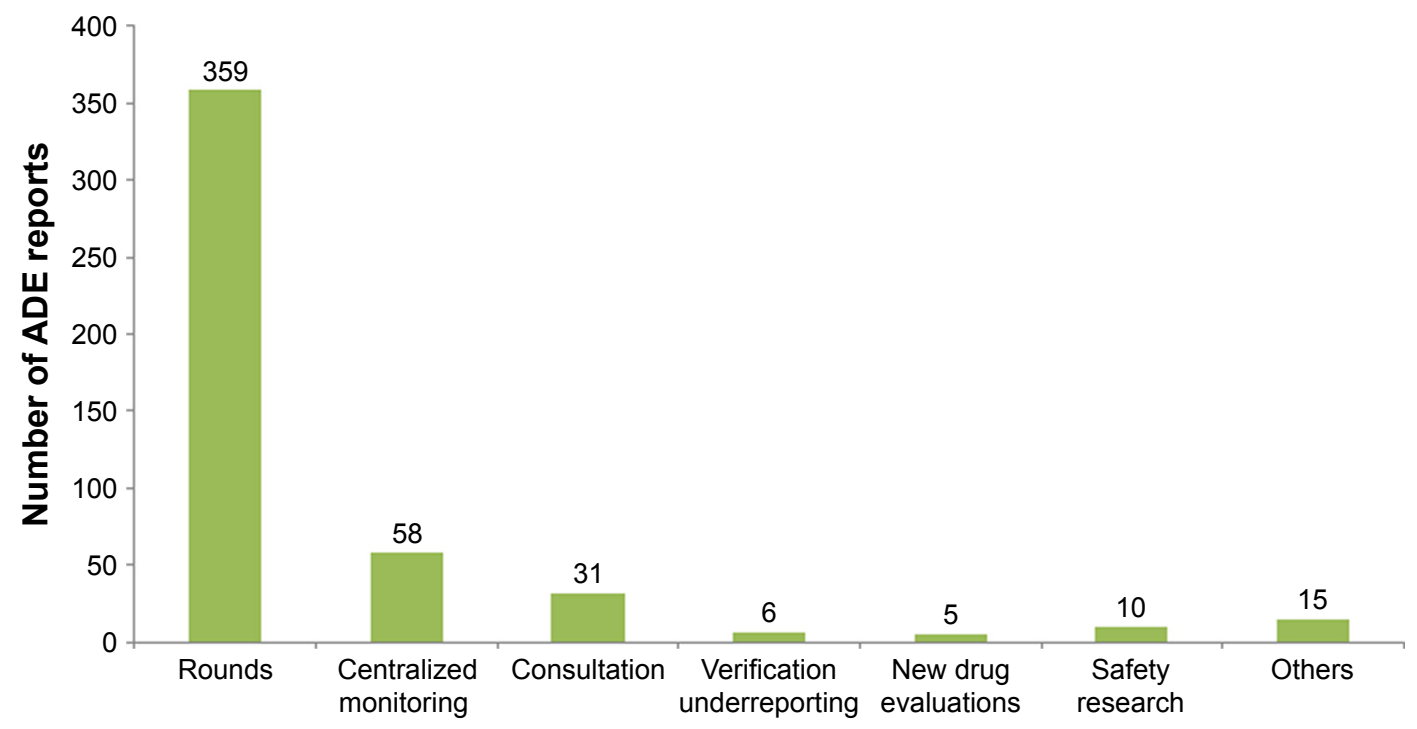

Figure 3 ADE source distribution reported by clinical pharmacists in 2017 . Abbreviation: ADE, adverse drug event. 
SAHZU's pharmacy department has explicitly included the ADE online report as part of the clinical pharmacists' work record. The online reports have a uniform format and can be downloaded, analyzed, and summarized at any time, which helps to improve the efficiency of the entire clinical pharmacist team and the use of past data, and ultimately promote the development of hospital pharmacovigilance. The last factor was the implementation of the new policy that all SAHZU's medical staff should give priority to reporting "new" and "serious" ADE. This change in strategy led to a significant reduction in the total number of reports in 2015 , but the proportion of "new" and "serious" ADE reports has increased significantly. The first three factors contributed to an increase in the number of ADE reports, while the last one led to a decline in the number of reports in 2015.

The proportion of "new" and "serious" ADE reports reflects the availability of the country's overall data on ADEs. ${ }^{13}$ However, it is often difficult for clinical staff to distinguish between the two types of ADE. In contrast, clinical pharmacists are familiar with the definition of "new" and "serious" adverse reactions. They can focus on monitoring and reporting of these two types of adverse reactions. The proportion of serious reports is an important indicator of the quality of adverse reaction databases. ${ }^{9}$ In general, the proportion of serious reports in developed countries accounts for more than $30 \%$ of the total number. In 2016, this ratio in China reached a record high, but it was only $\sim 7.5 \% .{ }^{14}$ Under the premise that the total number of reports increased rapidly, SAHZU maintained this proportion to grow continuously, because the main submitters of these reports are clinical pharmacists who can better distinguish and collect "serious" ADE reports. Our data showed that with the establishment of the "pharmacistled" model, the proportion of pharmacists reporting ADEs had increased gradually, and there was a significant difference between 2016 and 2017 compared to 2012. The proportion of severe ADE reports was high correlated with the proportion of ADEs reported by clinical pharmacists.

But the pharmacist-led ADE management model is not just about pursuing the number of ADE reports and the proportion of "new" and "serious" ADE reports. The model is aimed at pharmacovigilance and integrates ADE management into routine work of all aspects of clinical pharmacists. Simply monitoring and reporting ADEs only to meet national requirements should be avoided. This will result in the lack of support from hospital management and the lack of reporting enthusiasm from pharmacists due to the occupation of working hours. Blindly meeting the country's requirements is also prone to data fraud. Pharmacovigilance is not only about detecting adverse reactions to medicines but also about detecting/addressing all other drug-related safety problems. Data of 2017 showed that SAHZU's clinical pharmacists obtained ADE information from various types of daily pharmaceutical work in hospitals, and then the safety signals obtained from the abovementioned routine pharmacovigilance activities can be used to evaluate drug quality problems and identify inappropriate medication use to discover potential drug safety risks in the hospital. It helps clinical pharmacists to continuously improve drug-related work processes and establish an effective drug safety management model suitable for this hospital. For instance, ADE management pharmacists discovered five safety signals from ADE reports during the period from 2015 to 2017. After evaluation, the five signals were considered to be related to the dose, mode of administration, drug batch, solvent, and dispensing process, respectively. We have revised the abovementioned safety issues by modifying the Hospital Information System, changing drug batches, personnel education, and hospital internal network notifications.

According to the data from Center for Drug Reevaluation of CFDA, there were 1,616 tertiary hospitals registered by the CNCAM in 2015, of which 1,069 were not reported any ADE for the whole year. ${ }^{6}$ The data of the same year showed that $53 \%$ of Chinese ADEs were reported by physicians. It was obvious that the enthusiasm of physicians in tertiary hospitals to report ADE voluntarily was not high. Center for Drug Reevaluation of CFDA believed that the reasons for this mainly include tertiary hospitals' negligence in reporting ADEs, physicians' reluctance to spend time filling in reports, and concerns that such reports will result in medical disputes. ${ }^{6}$ Physician workload increased dramatically for Chinese physicians in the past 20 years. ${ }^{15}$ Compared with physicians in other hospitals, Chinese physicians in tertiary hospitals bear heavier workload, physical and mental stress. ${ }^{16}$ The specific work of reporting ADEs in Chinese medical institutions is generally carried out by clinical pharmacists. ${ }^{17}$ Their professional and work scope means that pharmacists know more about the importance of ADE monitoring and reporting than physicians, they are more familiar with the reporting process and monitoring priorities, and are less worried about medical disputes and other potential conflicts.

In addition, ADEs reported by clinical pharmacists have high degree of completeness and low modification rate. We found that $\mathrm{ADE}$ reports submitted by clinical pharmacists generally only need to review the completeness and modify 
simple writing errors, while reports from nurses and doctors may require supplementing more information. With the expansion of monitoring and management of ADEs in China, the requirements for the quality of reports have become higher at all levels of ADE monitoring. In addition to satisfying the true, complete, and accurate basic requirements, there is a higher requirement for the overall quality of cases. In the current reporting model, ADEs are reported by the clinical staff, and then the clinical pharmacist supplements the data and completes the report before reporting it to China's SRS. However, medical personnel do not necessarily report in a timely manner, and relevant information may not be completely recorded in the course of the disease, so information added later may not be complete and accurate. To increase the awareness and timely reporting rate of clinical staff for ADE reporting, SAHZU arranges lectures annually on ADEs at various levels such as the hospital and department levels, and clinical pharmacists also explain the specific reporting methods to the required departments and individuals. However, there are many physicians and nurses in tertiary hospitals, and their sources are relatively complex, including many training personnel and medical students, so it is difficult to maintain a high degree of awareness. The population of clinical pharmacists is relatively fixed, and the number is relatively small. Clinical pharmacists who have been trained by ADE management pharmacists can quickly master the ADE monitoring and reporting skill.

According to the requirement of the Ministry of Health, clinical pharmacists should help physicians to appropriately prescribe medicines for patients, and it is stipulated that all tertiary hospitals should have at least five full-time clinical pharmacists. ${ }^{17}$ Clinical pharmacist is uniquely trained to be able to impact medication safety at the individual patient level through medication management skills that are part of the clinical pharmacist's role but also to analyze the performance of medication processes and to lead redesign efforts to mitigate drug-related outcomes that may cause harm. ${ }^{18}$ Over the past several decades, the role of pharmacists has changed significantly, from a simple dispensing role to being more directly involved in optimizing patient drug treatment. At present, clinical pharmacists in China have generally participated in the direction of safe drug therapy, from reviewing prescriptions to monitoring drug effects. The routine work of SAHZU's clinical pharmacists includes ward rounds or consultation, patient education, therapeutic drug monitoring, ADE monitoring, drug evaluation and utilization research, and a training and management system.
However, clinical pharmacy in China is still in its infancy, and most pharmacists do not play a decisive role throughout the drug treatment process. ${ }^{19}$ Beginning with the drug adverse event management and pharmacovigilance that pharmacists are most "familiar is one of the better" methods to raise the professional level and clinical recognition of pharmacists. For instance, approximately one-third of SAHZU's pharmaceutical consultations are related to the analysis and treatment of ADEs. Inpatients usually need to use several or even dozens of drugs at the same time. When unexpected adverse clinical reactions occur, physicians need clinical pharmacists to help identify possible drug-related adverse events and suggest feasible treatments. These pharmaceutical consultations bring practical and effective help to the physicians' clinical work, which promoted the cooperative relationship between physicians and pharmacists and improved the professional image of pharmacists. All these national conditions have a positive impact on the establishment of pharmacist-led ADE management model.

\section{Weaknesses and future directions}

The model requires more clinical pharmacists. If the number of clinical pharmacists is low, the number of adverse reactions collected through rounds of inspection is too limited to reflect the situation in the whole hospital. A framework for the construction of the clinical pharmacist system has been established in China. There are now more than 20,000 clinical pharmacists in China and the numbers are growing gradually. ${ }^{17,20}$ In the future, the number of clinical pharmacists and the scope of the work will be larger, which is conducive to the development of clinical pharmacist-led management model.

The model has high requirements for the professional ability of pharmacists. Less competent pharmacists may have many misjudged or overreported cases. Monitoring agencies should strengthen the unified training of monitoring pharmacists to improve the ability of the pharmacists to evaluate and deal with ADEs.

However, the most important thing is the focuses of different medical staff regarding drug safety vary. Nurses are more concerned about medication errors, such as administering the wrong drugs or the wrong delivery time. Physicians are more concerned about rare serious adverse reactions, or frequently occurring ADEs that may be drug quality-related, whereas known common adverse reactions, even serious adverse reactions such as chemotherapy-induced grade 4 myelosuppression, are also considered as not necessary to 
report. From the point of view of drug safety management, the lack of reporting of the previously mentioned two sources of ADEs can result in management loopholes. Verifying the missing reports, intensive monitoring or clinical research by clinical pharmacists might compensate for this defect to a certain extent, but most of these are retrospective analysis and cannot be reported in real time. CHPS is expected to solve this problem partially in the future. CHPS was developed by CNCAM to collect and analyze information automatically extracted from sentinel hospitals. Its information platform was built on the information system of sentinel hospitals connected to the coordinating center of the program. Necessary structured information is extracted from the Hospital Information System and evaluated by clinical pharmacists of sentinel hospitals and then is uploaded to CNCAM, the coordinating center, to enrich data resources of the SRS system. ${ }^{3}$

\section{Conclusion}

China has become the world's second largest pharmaceutical consumer market. ${ }^{21}$ The rapid improvement of China's pharmaceutical market in the international arena raises higher requirements and challenges for Chinese pharmacovigilance. As the major source of national security reports, medical institutions need to constantly practice and explore work patterns that are suitable for the conditions of each hospital. The pharmacist-led pharmacovigilance working model significantly increased the quantity and quality of ADE reporting in SAHZU and promoted pharmacovigilance. This model is worth developing in Chinese tertiary hospitals and the following hospitals, as the physicians working there spend little time and energy on ADE reporting or the cost of physicians is high, while the clinical pharmacist team has strong professional skills.

\section{Acknowledgments}

This study was supported by the research grants from the foundation from the Zhejiang Provincial Natural Science Foundation of China (LY16H310001, LY15H310006), Zhejiang Province Medical and Health Research Program (2015KYB187), and the Department of Education of the Zhejiang Province of China (Y201431669, Y201328670).

\section{Disclosure}

The authors report no conflicts of interest in this work.

\section{References}

1. World Health Organization. The Importance of Pharmacovigilance Safety Monitoring of Medicinal Products; 2002. Available from: http:// apps.who.int/medicinedocs/en/d/Js4893e/. Accessed June 14, 2018.
2. Fang H, Lin X, Zhang J, et al. Multifaceted interventions for improving spontaneous reporting of adverse drug reactions in a general hospital in China. BMC Pharmacol Toxicol. 2017;18(1):49.

3. Li X, Li H, Deng J, et al. Active pharmacovigilance in China: recent development and future perspectives. Eur J Clin Pharmacol. 2018; 74(7):863-871.

4. Dong D, Liu CL. The Revelation of Study on Drug Manufacture Post Marketing Surveillance System in the United States. Chinese Journal of pharmacovigilance. 2013;10(8):456-459.

5. Food and Drug Administration. FAERS Reporting by Healthcare Providers and Consumers by Year; 2015. Available from: http://www.fda. gov/Drugs/GuidanceComplianceRegulatoryInformation/Surveillance/ AdverseDrugEffects/ucm070456.htm. Accessed June 14, 2018.

6. Shen L, Liu W, Guo X. Ideas on trend of ADR monitoring model in China. Chinese Journal of pharmacovigilance. 2017;14(5):295-297.

7. Xiao C, Li Y, Baytas IM, Zhou J, Wang F. An MCEM Framework for Drug Safety Signal Detection and Combination from Heterogeneous Real World Evidence. Sci Rep. 2018;8(1):1806.

8. Kreimeyer K, Menschik D, Winiecki S, et al. Using Probabilistic Record Linkage of Structured and Unstructured Data to Identify Duplicate Cases in Spontaneous Adverse Event Reporting Systems. Drug Saf. 2017; 40(7):571-582.

9. National Health and Family Planning Commission of the People's Republic of China. Drug Adverse Reaction Reporting and Monitoring Management Measures (Ministry of Health of People's Republic of China Order No. 81); 2011. Available from: http://www.moh.gov.cn/ mohzcfgs/pgz/201105/51770.shtml. Accessed June 15, 2018.

10. Huang YL, Moon J, Segal JB. A comparison of active adverse event surveillance systems worldwide. Drug Saf. 2014;37(8):581-596.

11. Pal SN, Olsson S, Brown EG. The monitoring medicines project: a multinational pharmacovigilance and public health project. Drug Saf. 2015;38(4):319-328.

12. Rolfes L, van Hunsel F, Caster O, Taavola H, Taxis K, van Puijenbroek E. Does patient reporting lead to earlier detection of drug safety signals? A retrospective comparison of time to reporting between patients and healthcare professionals in a global database. Br J Clin Pharmacol. 2018; 84(7):1514-1524.

13. Ma ZP. Research Progress of Individual Case Safety Report and Periodic Safety Update Reports. Journal of Shenyang Medical College. 2016;18(4):302-304.

14. China Food and Drug Administration. Annual Report on National Monitoring of Adverse Drug Reactions. 2017-04-28; 2016. Available from: http://www.sda.gov.cn/WS01/CL0844/172167.html. Accessed June 14, 2018.

15. Fu Y, Schwebel D, Hu G. Physicians' Workloads in China: 1998-2016. Int J Environ Res Public Health. 2018;15(8):1649.

16. Sun J, Ma J, Hu G, et al. Welfare, wellness, and job satisfaction of Chinese physicians: A national survey of public tertiary hospitals in China. Int J Health Plann Manage. 2017;32(3):270-284.

17. Chen B, Huang JJ, Chen HF, Xu BM. Clinical pharmacy service practice in a Chinese tertiary hospital. Drug Metab Pers Ther. 2015;30(4):215-230.

18. Mansur JM. Medication Safety Systems and the Important Role of Pharmacists. Drugs Aging. 2016;33(3):213-221.

19. Rao Y, Zhao Q, Zhang X, Yang H, Lou Y, Zhang X. Current status and future prospects of the development of clinical Pharmacy in China: A SWOT analysis. Pak J Pharm Sci. 2016;29(2):415-421.

20. Chinese Hospital Association. Notice on Further Strengthening the Construction of Clinical Pharmacist System. 2016-11-25. Available from: http://www.cha.org.cn/plus/view.php?aid=15129. Accessed June 20, 2018.

21. Central People's Government of China. China has become the second largest pharmaceutical consumer market in the world; 2017. Available from: http://www.gov.cn/xinwen/2017-07/12/content_5209966.htm. Accessed June 21, 2018. 
Therapeutics and Clinical Risk Management

Dovepress

\section{Publish your work in this journal}

Therapeutics and Clinical Risk Management is an international, peerreviewed journal of clinical therapeutics and risk management, focusing on concise rapid reporting of clinical studies in all therapeutic areas, outcomes, safety, and programs for the effective, safe, and sustained use of medicines. This journal is indexed on PubMed Central, CAS,

EMBase, Scopus and the Elsevier Bibliographic databases. The manuscript management system is completely online and includes a very quick and fair peer-review system, which is all easy to use. Visit http://www.dovepress.com/testimonials.php to read real quotes from published authors.

Submit your manuscript here: http://www.dovepress.com/therapeutics-and-clinical-risk-management-journal 